\title{
AUTONOMOUS OSCILLATIONS WITHOUT EXTERNAL POWER SUPPLY IN A MEMS OSCILLATOR THROUGH INTERNAL RESONANT MODE COUPLING
}

\author{
C. Chen ${ }^{1}$, D.H. Zanette ${ }^{2}$, D.A. Czaplewski ${ }^{1}$, and D. López ${ }^{1 *}$ \\ ${ }^{1}$ Center for Nanoscale Materials, Argonne National Laboratory, Lemont, Illinois, USA \\ ${ }^{2}$ Centro Atomico Bariloche, Río Negro, Argentina
}

\begin{abstract}
Oscillators are systems that produce stable periodic signals without external frequency reference. A typical oscillator consists of a resonator that determines the output frequency, and an external sustaining feedback scheme to replenish the energy loss due to the dissipation from the resonator. Here we demonstrate that, by driving the constituent resonator to the internal resonance (IR) condition, where two different vibrational modes of the same resonator are coupled commensurately, there is efficient coherent energy exchange between the two modes. This strong coupling allows the observed mode to draw stored energy from the coupled mode, hence maintaining both its oscillation amplitude and frequency without an external power supply.
\end{abstract}

\section{INTRODUCTION}

Self-sustained oscillators that autonomously create prescribed frequencies are critical in modern technology, with applications such as time keeping, communication and navigation [1]. A typical oscillator consists of a mechanical resonator that determines the output frequency of the oscillator and a sustaining feedback element. During the oscillations, the mechanical resonators inevitably dissipate energy to the environment, through mechanisms such as viscous damping, clamping losses, and thermoelastic dissipations. In order to compensate such losses and maintain the vibrational amplitude, the sustaining element draws energy from external sources and supplies the energy to the resonator. Examples of sustaining elements include wound spring and electronic amplifiers. At steady state, the energy influx equals the energy dissipation, hence satisfying the well-known Barkhausen criterion, and the oscillator produces a stable, continuous signal.

In this classic design of oscillators, the mechanical resonator is operated in the linear regime, in which the resonant frequency $f$ is independent of the vibrational amplitude, $A$. However, as the critical dimensions of the resonator shrink toward micro- and nano-meter scales as in the cases of micro- and nano-electromechanical systems (MEMS and NEMS), the size of the linear regime also decreases accordingly. For example, the critical vibrational amplitude of a doubly clamped resonator scales as $L / Q^{0.5}$, where $L$ is the resonator length and $Q$ is the quality factor [2]. When the oscillation amplitude is above this critical value, (also known as onset of nonlinearity), the resonant frequency becomes dependent on the vibrational amplitude, known as the $A-f$ effect [3]. Since the onset of nonlinearity diminishes for MEMS and NEMS, small levels of excitation, or even mere thermomechanical noise can put the resonators into the nonlinear regime [4]. Recent investigations of nonlinear MEMS and NEMS oscillators have demonstrated improved frequency stability [5], enhanced synchronization range [6], and superior performance in energy harvesting applications [7].

In this study, we exploit the $A-f$ effect in a high- $Q$ silicon MEMS oscillator to invoke internal resonance (IR), where the resonant frequency of the in-plane mode is tuned to match one-third of the resonant frequency of its own torsional mode. Previously we have shown reduced phase noise [8] of the oscillator at IR; here we explore the dynamical response at IR, and find anomalous ring-down behavior of the in-plane mode where it keeps oscillating without external energy supply for as long as 227 milliseconds. As a practical demonstration of this discovery, we show continuous operation of the oscillator when the external sustaining circuit is only engaged intermittently.

\section{DESCRIPTION OF INTERNAL RESONANCE (IR)}

For a nonlinear, $N$-degree-of-freedom system, in which each degree of freedom represents a vibrational mode with a unique linear resonant frequency, important cases emerge as two or more resonant frequencies are commensurable or near commensurable. Internal resonance describes the situation when such commensurable relationship agrees with the order of nonlinearity of the system [9]. The hallmark of IR is that, if energy is initially imparted to only one mode, there will be continuously energy exchange among all the modes involved in the IR, while all the modes will also dissipate energy to the environment due to their respective damping.

Let us consider a system that consists of 2 modes ( $x$ and $y$ ) as follows:

$$
\begin{aligned}
& \ddot{x}+Q^{-1} \dot{x}+x+\beta x^{3}=f_{d} \cos \left(\omega_{d} t\right)+J_{y} y \\
& \ddot{y}+q^{-1} y+\Omega_{y}^{2} y=J_{x} x
\end{aligned}
$$

Here $Q$ and $q$ are the quality factors (inverse of dissipations) of each mode, respectively. The linear resonant frequencies of mode $x$ and $y$ are 1 and $\Omega_{y}$. We only consider cubic nonlinearity in mode $x$ (Duffing-type), with corresponding nonlinear coefficient of $\beta$. We also only consider linear couplings between the two modes, with strength of $J_{y}$ and $J_{x}$. Addtionally, only mode $x$ is being driven externally, with forcing amplitude of $f_{d}$ and frequency of $\omega_{d}$; therefore, external energy is only injected to mode $x$. If there is no inter-mode coupling $\left(J_{y}=J_{x}=0\right)$, then mode $x$ is a simple driven nonlinear resonator, and mode $y$ is a damped, undriven linear resonator.

Since the order of nonlinearity in this system is 3 , IR happens if $\Omega_{y} \approx 1 / 3$ or 3 . However, due to the nonlinear nature of mode $x$, namely, the $A-f$ effect, the resonant frequency of mode $x$ can be greater than 1 for positive $\beta$ and less than 1 for negative $\beta$. Therefore, it is possible to encounter the IR condition for values of $\Omega_{y}$ different from $1 / 3$ or 3 . For exmple, if $\beta$ is positive, the $A-f$ effect will result in the resonant frequency of mode $x$ being larger than 1 , hence IR can occur for $\Omega_{y}>3$ as long as $\Omega_{y}$ equals three times the nonlinear resonant frequency of mode $x$. To demonstrate the possibility of IR under such a condition, we perform long-time numerical integration of equation (1), with $Q=q=50, \beta=1, \Omega_{y}=$ 3.3, and $J_{y}=2, J_{x}=1$. The simulated results are shown in Fig. 1, for different values of $f_{d}$ and $\omega_{d}$. For each simulation, the value of $f_{d}$ is fixed, and the value of $\omega_{d}$ is swept upward.

The steady state amplitude of mode $x$, shown in Fig. 1a, displays the normal frequency tuning effect for small $f_{d}$, where the resonant frequency $\omega_{x}-$ at which point the amplitude reaches maximum value - increases with $f_{d}$. However, for intermediate values of $f_{d}$, we find that $\omega_{x}$ becomes less dependent on $f_{d}$ and stays around 1.1 , which is $1 / 3$ of $\Omega_{y}$. Therefore, we conclude that IR is reached, and the surpressed frequency tuning is one of its characteristics. The surpressed frequency tuning can be understood 
as follows: when more energy is imparted to mode $x$ (as one increases the excitation amplitude $f_{d}$ ), this energy is transferred to mode $y$ through IR, hence the amplitude (and frequency) of mode $x$ remains nearly constant. The relation between $\omega_{x}$ and $f_{d}$ is better presented in Fig. 2, where we clearly observe a plateau in $\omega_{x}$. At IR, we also find a sudden increase in the amplitude (hence kinetic energy) of mode $y$, shown in Fig. 1b. Since mode $y$ can only obtain energy through coupling to mode $x$, such an abrupt increase in mode $y$ 's energy illustrates the enhanced energy transfer between the two modes at IR. For large $f_{d}$, which pushes $\omega_{x}$ above 1.1, the normal frequency tuning resumes, and the IR condition is no longer active.
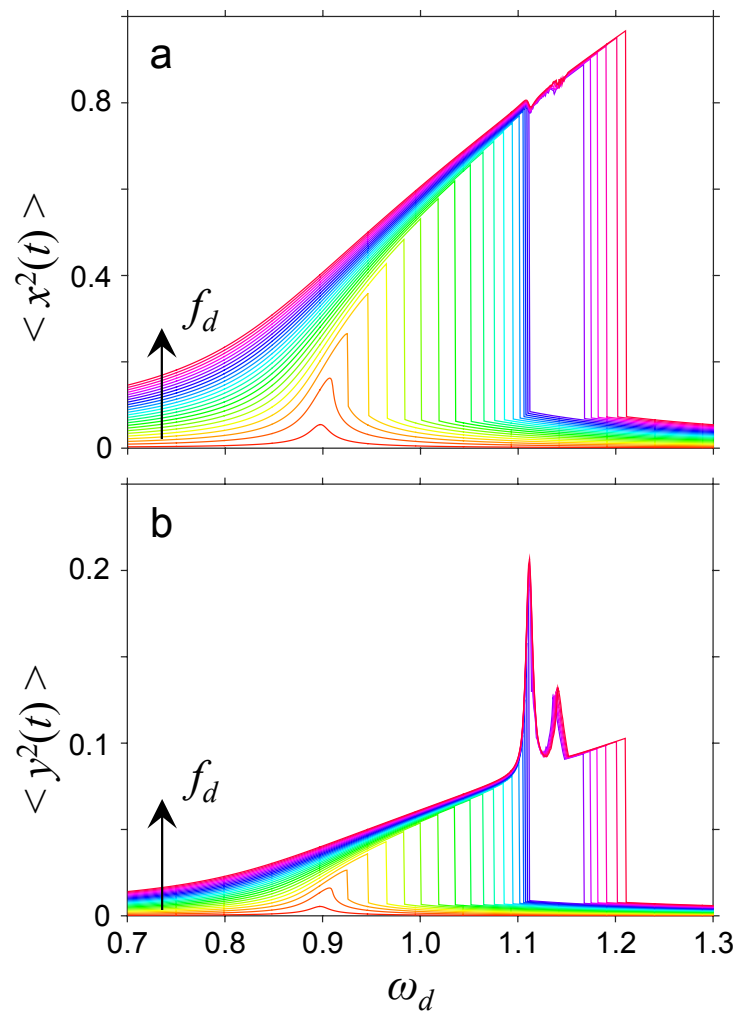

Figure 1: Steady state amplitudes of mode $x$ and mode $y$, obtained from long-time numerical integrations of equation (1). The forcing amplitude $f_{d}$ is increased from 0.001 to 0.051 , with 0.002 step size. The linear resonant frequency for mode $x$ is downshifted from 1 to 0.9, due to the coupling to mode $y$.

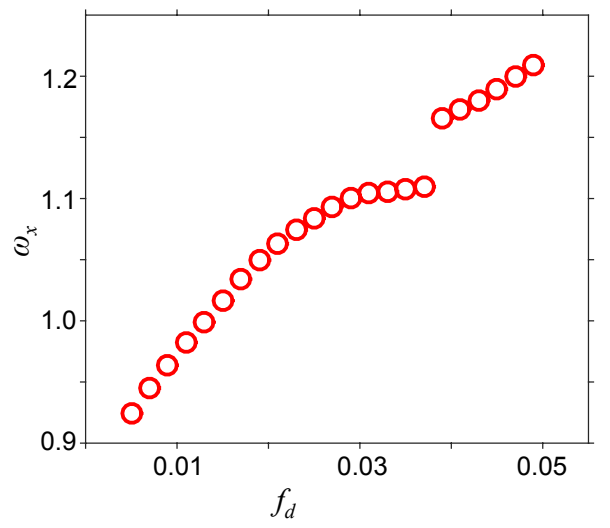

Figure 2: Resonant frequency of mode $x, \omega_{x}$, as a function of forcing amplitude $f_{d}$. Data is extracted from the simulation results shown in Fig. 1a. The resonant frequency $\omega_{x}$ is defined where the amplitude of mode $x$ reaches its maximum value.

\section{EXPERIMENTAL EVIDENCE OF IR}

We use single crystalline, doubly clamped silicon MEMS resonators to experimentally investigate the characteristics of IR. The devices are fabricated from the SOIMUMPS process from MEMSCAP. A typical device is shown in the inset of Fig. 3. The MEMS resonator is excited electrostatically with interdigitated comb electrodes, and the mechanical motion is transduced electrostatically with another set of comb electrodes. We use COVENTOR to determine the mode shapes and frequencies of the first three vibrational modes, as summarized in Table 1 . The discrepancy between the simulated and measured resonant frequency most likely results from variations in the fabrication process.

Table 1: Simulated and measured linear resonant frequency for the first three vibration modes. The linear resonances for the out-of-plane and the torsional mode are measured with an optical interferometer.

\begin{tabular}{|c|c|c|}
\hline Mode shape & $\begin{array}{c}\text { Simulated } \\
\text { frequency (kHz) }\end{array}$ & $\begin{array}{c}\text { Measured } \\
\text { frequency (kHz) }\end{array}$ \\
\hline In-plane & 55.734 & 61.570 \\
\hline Out-of-plane & 179.071 & 159.832 \\
\hline Torsional & 201.082 & 194.636 \\
\hline
\end{tabular}

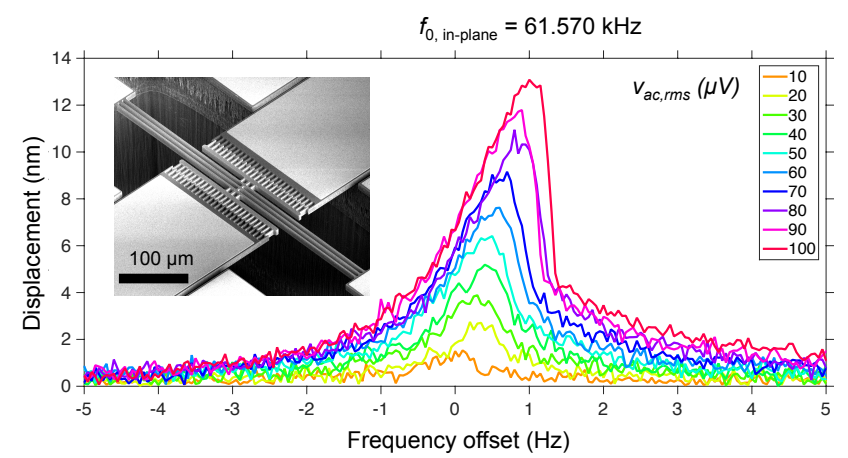

Figure 3: Vibrational amplitude of the in-plane mode of the MEMS resonator with different ac excitation. The applied DC bias voltage is $7 \mathrm{~V}$. The amplitude is measured with a lock-in amplifier (Zurich Instrument HF2LI). Inset: Scanning Electron microscopic image of the doubly clamped silicon beam.

The mode of interest (mode $x$ ) here is the in-plane vibrational mode, with linear frequency of $61.570 \mathrm{kHz}$ and quality factor of 120,000 , measured in vacuum. The high quality factor results in an onset of nonlinearity of about $10 \mathrm{~nm}$ (Fig. 3), occurring with small alternative current (ac) excitation just below $100 \mu \mathrm{V}$. This small onset of nonlinearity allows for large resonant frequency tuning through the $A-f$ effect when the drive voltage is increased.

In order to identify the presence of IR in our MEMS resonator, we turn to closed-loop, oscillator-like schematics, where the MEMS resonator serves as the frequency determining element. This is equivalent to the situation of replacing the external excitation term $f_{d} \cos \left(\omega_{d} t\right)$ in equation (1) with a feedback excitation term. Specifically, we employ two types of oscillator architectures. In design A (see Fig. 4), the feedback loop includes 
electronic amplifiers and a phase-locked loop (PLL), and the amplitude of the feedback excitation is controlled by the output of the PLL. In this configuration, as we increase the forcing amplitude, we find that the resonant frequency shifts up steadily from $61.6 \mathrm{kHz}$, before "accumulating" around $64.9 \mathrm{kHz}$, which coincides with $1 / 3$ of the resonant frequency $(194.6 \mathrm{kHz})$ of the torsional mode (mode $y$ ). Therefore, the IR between the in-plane mode and torsional mode is clearly observed. The linear resonant frequency of the torsional mode is measured separately with an optical interferometer. In design B (see Fig. 5), we replace the PLL with a phase shifter, and the stable oscillations are achieved by the $A-f$ effect [11]. In either case, we have observed IR manifested itself within a wide range of control parameters (driving force for design A and phase-delay for design B). For design circuit B, there is a regime where multiple oscillation frequencies exist for the same phase-delay $\Delta$ (for $\Delta$ around $40^{\circ}$ ), this is due to the multi-value nature of the nonlinear resonator [9]. We will use design $\mathrm{B}$ for the rest of the characterizations because it has simpler closed loop components.

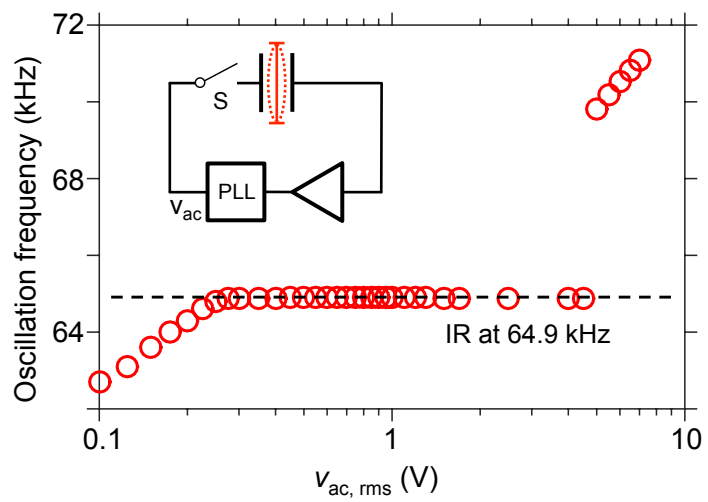

Figure 4: Oscillator design A, in which the feedback force is controlled by the output amplitude of the PLL. The IR persists for a wide range of driving amplitudes. Note that the $x$-axis is in logarithm scale.

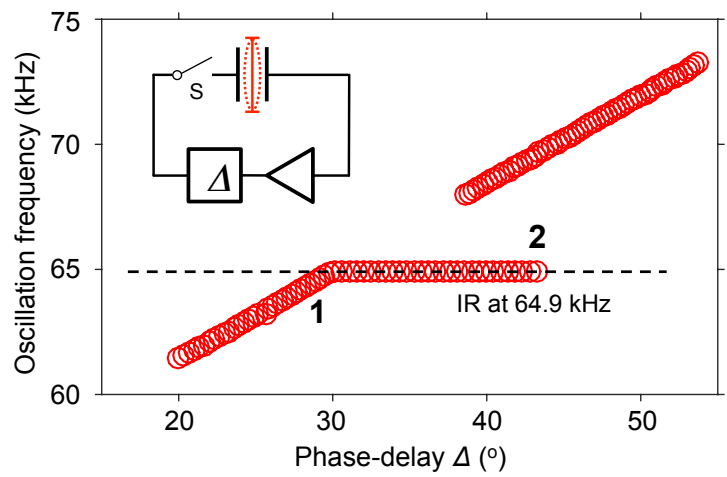

Figure 5: Oscillator design B, in which the feedback loop consists of electronic amplifiers and phase-shifter. The oscillation frequency is controlled by the phase-delay $\Delta$ introduced by the phase shifter. The IR is characterized as the regime where the oscillation frequency is insensitive to $\Delta$.

\section{DYNAMICAL RESPONSE AT IR}

In order to study the dynamical behavior of IR, especially the energy exchange between the two modes, we turn to explore the temporal response at the IR condition. We perform ring-down measurement on the in-plane mode to examine its dynamical behavior. After the MEMS oscillator reaches steady state, we remove the feedback excitation by disengaging the switch $\mathrm{S}$, and record the transient revolution of its amplitude with a digital oscilloscope (Tektronix, DPO5204, sampling rate of $2 \mathrm{MSample} / \mathrm{second}$ ). If the oscillator is positioned outside IR (point 1 in Fig. 5), the vibrational amplitude decays immediately after the external excitation is removed (Fig. 6), with a decay rate dictated by the quality factor. In the case of nonlinear oscillators, the instantaneous frequency will also decay toward the linear resonance due to the $A-f$ effect [10].
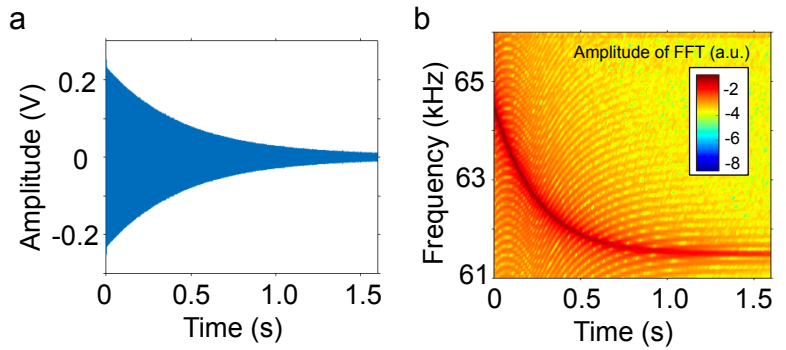

Figure 6. Ring-down responses of the nonlinear oscillator outside IR. (a) Measured temporal evolution of the oscillation amplitude after the excitation is removed. (b) Temporal frequency response of the oscillation. The power spectrum at each nominal time instance $t_{i}$ is obtained by performing non-overlapping fast Fourier transform (FFT) of the time domain data (a) in a narrow window around $t_{i}$. a

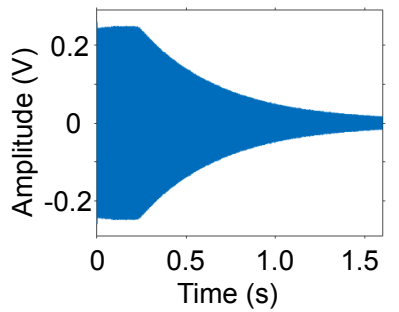

b

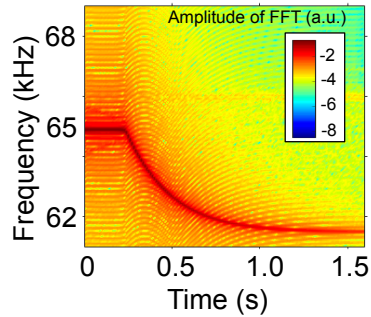

Figure 7. Ring-down responses of the nonlinear oscillator at IR. (a) Measured temporal evolution of the oscillation amplitude after the excitation is removed, showing a period of 227 milliseconds where the amplitude does not decay. (b) Temporal frequency response of the oscillation, where the oscillation frequency stays at $64.9 \mathrm{kHz}$ during the first 227 milliseconds, before expected exponential decay towards linear resonance.

However, when the oscillator is operating at the IR condition (point 2 in Fig. 5), the vibrational amplitude does not decay for as long as 227 milliseconds (Fig. 7a), after which the amplitude starts to decrease as in previous ring-down experiments. Moreover, during this period of time, the oscillation frequency stays constant and stabilized at $64.9 \mathrm{kHz}$ (Fig. 7b). The origin of this unexpected behavior of nearly constant amplitude and stabilized frequency demonstrate the presence of the coherent energy transfer at IR: at steady state (before ring-down), extra mechanical energy is transferred to and stored in the torsional mode through IR. Once the external power supply is removed as the ring-down occurs, the in-plane mode starts to receive energy from the torsional mode, 
thus compensating the energy loss due to its own energy dissipation. Once the energy of the torsional mode is depleted, the in-plane mode will begin normal ring-down as a nonlinear oscillator.
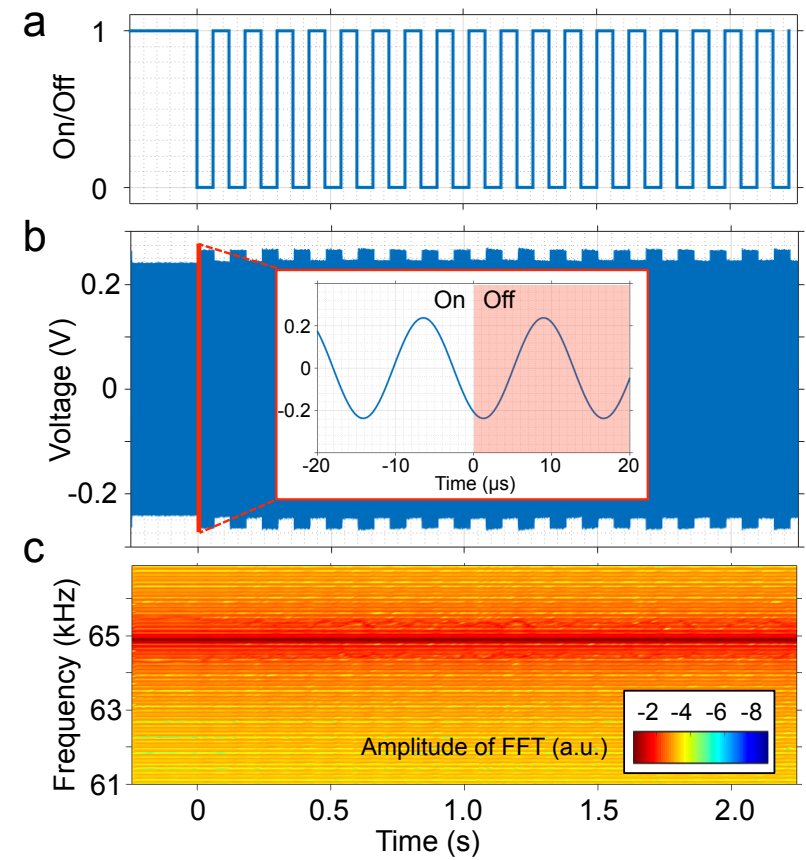

Figure 8. Continuous oscillations with a 50\% duty cycle. (a) The duty cycle profile that controls the switch $S$. The cycle period is 120 milliseconds. (b) Measured oscillation amplitude, where the slight jumps during the switching events are caused by the impedance mismatch of the RF switch used to control the feedback loop. The inset shows zoomed-in oscillation around $t=0 \mathrm{~s}$, where no sign of discontinuity is observed. (c) Temporal frequency spectrum during the cycles.

During this 227 millisecond period, the MEMS oscillator runs solely on the energy stored in the coupled mechanical mode, and continues to operate as when it was in the closed loop. Therefore it allows the external sustaining circuitry to be switched off intermittently. As a demonstration, we apply an on/off signal with a $50 \%$ duty cycle and a period time of 120 milliseconds to the switch that controls the feedback loop (S in Fig. 5), and record the temporal response of the oscillator, as shown in Fig. 8. Over the period of more than 2 seconds that is being recorded, the vibrational amplitude doesn't show any sign of decay during the "off" period. Furthermore, there is no discontinuity in the waveform between the "on/off" events, shown as the inset of Fig. $8 \mathrm{~b}$. The slight jumps of the overall envelop of the vibrational amplitude during the switching events are caused by the impedance mismatch of the switch S. As a direct result of the constant amplitude, the oscillation frequency during the whole period also stays constant (Fig. 8c). This observation highlights the advantage of the IR for practical applications: not only can it improve the frequency stability of the oscillator [5], the usage of pulse-like patterns will also enable new power-efficient protocol for MEMS oscillators.

\section{CONCLUSION}

We provide the first demonstration of an autonomous oscillator that can be operated without an external power supply while keeping a finite amplitude and stabilized frequency. We use a nonlinear silicon MEMS oscillator that is driven to the condition of IR where there is strong mutual energy transfer between the coupled modes (in-plane mode and torsional mode). By characterizing the ring-down responses of the in-plane mode outside and inside IR, we show that at the IR condition, the coherent energy transfer from the torsional mode to the in-plane mode is able to keep the latter oscillating with constant amplitude and stabilized frequency for as long as 227 milliseconds. These autonomous oscillators show potential to be used as timing references for low-power applications or in extreme environments with limited power supply. Our findings therefore enable a new paradigm for harnessing nonlinearities in MEMS and NEMS.

\section{ACKNOWLEDGEMENT}

Use of the Center for Nanoscale Materials at the Argonne National Laboratory was supported by the U.S. Department of Energy, Office of Science, Office of Basic Energy Sciences, under Contract No. DE-AC02-06CH11357. We thank D. Antonio for helpful discussions.

\section{REFERENCES}

[1] C. Audoin and B. Guinot, The measurement of time: time, frequency and the atomic clock, Cambridge University Press, 2001.

[2] K. Ekinci, M. Roukes, "Nanoelectromechanical Systems", Review of Scientific Instruments, 76, 061101 (2005).

[3] M. Agarwal, et al., "Optimal drive condition for nonlinearity reduction in electrostatic microresonators." Applied Physics Letters 89.21 (2006): 214105.

[4] J. Gieseler, L. Novotny, and R. Quidant, "Thermal nonlinearities in a nanomechanical oscillator", Nature Physics, 9, 806 (2013).

[5] D. Antonio, D. Zanette, and D. López, "Frequency stabilization in nonlinear micromechanical oscillators", Nature Communications 3 (2012): 806.

[6] D. A. Czaplewski, et al., "Enhanced synchronization range from non-linear micromechanical oscillators", IEEE Solid-State Sensors, Actuators and Microsystems (TRANSDUCERS), 2015.

[7] F. Cottone, H. Vocca, and L. Gammaitoni. "Nonlinear energy harvesting”, Physical Review Letters 102.8 (2009): 080601.

[8] D. A. Czaplewski, et al., "Phase noise reduction in an oscillator through coupling to an internal resonance", Solid-State Sensors, Actuators and Microsystems Workshop (Hilton Head), 2014

[9] A. Nayfeh, and D. Mook, Nonlinear oscillations. John Wiley \& Sons, 2008.

[10] P. Polunin, et al., "Characterizing MEMS nonlinearities directly: The ring-down measurements." IEEE Solid-State Sensors, Actuators and Microsystems (TRANSDUCERS), 2015.

[11] C. Chen, et al., "A self-sustained mechanical oscillator with linear feedback", submitted.

\section{CONTACT}

*D. López,dlopez@anl.gov 